\title{
La importancia de los materiales en las viviendas de muy bajo coste
}

\begin{abstract}
RESUMEN
El texto que sigue se encuadra en el contexto de intentar enmarcar la importancia considerable del capítulo de los materiales de construcción en el subsector de las viviendas de muy bajo coste en los Países en Vías de Desarrollo en general, y en Latinoamérica en particular. El autor trata de cuantificar, en forma global, la repercusión de los materiales en varios supuestos y como ésta aumenta al disminuir - prácticamente desaparecer-las partidas de instalaciones y acabados.
\end{abstract}

De conformidad y como consecuencia de lo anterior, el autor se ocupa de la necesidad de emplear materiales autóctonos y de apoyar la investigación y el desarrollo tenológico que ponen énfasis en la utilización de materiales alternativos y la reutilización de desechos agro-industriales.

Formando parte del Capítulo $V$ que se reproduce, se contempla un apartado específico con el título "La Industria de Materiales de la Construcción" que hemos omitido, remitiendo al lector interesado al n. ${ }^{2} 198$ de esta Revista, donde se publicó en primicia dicho trabajo.

\begin{abstract}
$S U M M A R Y$
This text fits into the context of the attempt to emphasize the considerable importance of the factor of the construction materials in the subsection of low cost housing in Developing Countries in general and in Latin America in particular. The author tries to quantify, in a glohal manner, the repercussion of the materials through different assumptions and to show how it increases as the sections of installations and finishes diminish -in fact, practically disappear-.
\end{abstract}

In accordance with this and as a consequence, the author discusses the necessity to use autochthonous materials and to support the research and technological development which lay stress on the use of alternative materials and the re-use of agro-industrial waste.

As a part of the Chapter $V$ reproduced here, a special Section entiteled "The Industry of Construction Materials" is contemplated, a section which we omitted and which can be found in $n .{ }^{2} 198$ of this Quarterly where it was originally published.

\section{NOTA PREVIA}

El trabajo que sigue es reproducción parcial del Capítulo V del libro "CONTRA EL HAMBRE DE VIVIENDA. Soluciones Tecnológicas Latinoamericanas", recientemente publicado por la Editorial ESCALA de Bogotá (Colombia) y presentado por el autor ante la "I. a Reunión de Ministros y Responsables Máximos de Vivienda y Urbanismo de América Latina" reunidos en la CEPAL (Santiago de Chile). La presentación en España tendrá lugar en la Casa de América el 26 de noviembre, y correrá a cargo de los Profesores José A. Fernández Ordóñez y Antonio Fernández Alba.

La distribución en España del libro se ha encomen- dado a la Editorial Polifemo (Avda. de Bruselas, 44; 28028 Madrid) y a la librería de la Casa de América.

\section{V.1. INTRODUCCIÓN}

Abordar algunas de las facetas del coste de ejecución de las viviendas de interés social, nos parece que requiere, cuando menos, una serie de precisiones que traten de enmarcar de qué economías, de qué construcciones y en qué contexto hablamos.

Trataremos de exponer las ideas básicas, a través del claro-oscuro de las profundas diferencias NorteSur, desarrollo-subdesarrollo o, si se quiere, entre 
mercado de oferta de viviendas versus la situación de prácticamente todos los países en vías de desarrollo: infraviviendas, hacinamiento, promiscuidad en proporciones masivas, etc.

Un primer aspecto importante para abordar, es el de la relativamente alta influencia de los materiales en los países en vías de desarrollo, en el coste de la construcción de las viviendas, en relación con los países desarrollados. Hay quienes extrapolando llegan a concluir, a nuestro criterio exageradamente, que el problema de la vivienda en los P.V.D. ( $\left.{ }^{*}\right)$ es antes que nada un problema de materiales de construcción.

Muchas veces, la lógica de los costes del Sur no es reflejo cabal de lo que ocurre en el Norte, sino una imagen distorsionada y grotesca como la de los espejos mágicos. Dos ejemplos: el cemento y el asfalto. H. Houben (1) cita trabajos del Banco Mundial en los que se constata que en Guinea el precio del cemento para los constructores es de 2,30 veces superior al pagado por el Estado y que en el mercado negro del mismo país, el precio estable es 7,9 veces mayor. El precio en Alemania de la tonelada de asfalto puede oscilar entre 1 y 3 según el país a que se destine. Otro trabajo siguió la pista al trascurrir de una tonelada de cemento belga, demostrando que llegaba a los usuarios de provincias de África hasta ocho veces más caro que su precio actual en la Capital de Europa.

Estos hechos explican en parte el porqué de la alta repercusión del cemento en el coste de construcción de la vivienda que suele sobrepasar el $15 \%$, e incluso llega al $25 \%$ en los P.V.D., mientras que en el Norte, se acerca con dificultad a la zona del $3 \%$. Estos desequilibrios, lejos de tender hacia una mejora se agravan, y a ello se refiere el Dr. Ramachandran, Director de UNCH-HABITAT cuando afirma (2) que "la importante cuenta de los P.V.D. en materiales de construcción ha crecido extraordinariamente desde los 6,1 billones de \$USA en 1970 hasta los 49,4 de 1980" o cuando aporta el dato terrorífico de que "en algunos países de África, más del $90 \%$ de los pagos del valor de los materiales de construcción que se utilizan en el sector formal corresponden a importaciones".

Aun siendo contundentes las razones argumentadas en pro de la importancia de los materiales, en su relación con la vivienda social, hay otra razón que en no pocas ocasiones nos lo recuerda a lo largo y ancho de América: las capas populares, los marginales... ahorran en materiales. Ellos ni lo entienden, ni tienen acceso al ahorro tradicional, pero sí saben del valor de un centenar de ladrillos, de una puerta o de un aparato sanitario.

(*) P.V.D.: Abreviatura de Paises en Vias de Desarrollo.

\section{V.2. LOS MATERIALES EN EL NORTE Y EN EL SUR}

Analizando algunos datos cuantitativos -forzosamente globales - es factible ver la diferencia de ópticas Norte-Sur ante la importancia de los materiales y la existencia de factores retardadores del desarrollo de la tecnología en el subsector vivienda. Este concepto queda meridianamente claro en la siguiente cita: "Una consideración importante, es el hecho de que en la composición del costo final de la vivienda producida formalmente a través del Servicio Federal de la Vivienda (SFH), algunas partidas como el suelo y la financiación presentan una tendencia a figurar con importancia creciente: estos factores, en cierto modo, han desestimulado el progreso de la tecnología de la edificación" (3).

La idea tomada del "Programa Integrado para Desenvolvimiento de Asentamientos Humanos", elaborado por el CNPq de Brasil, ratifica cómo los factores externos al capítulo construcción, son importantes en la conformación del precio final de la vivienda, esencialmente: suelo y financiación, actuando de hecho como retardadores del factor tenológico de la construcción de viviendas, especialmente en el sector formal.

Ratificando la sutil apreciación brasileña, que compartimos cabalmente, parece oportuno presentar una situación, posiblemente extrema, pero típica del Norte. Se trata de los valores medios -elaborados por la Revista Alfoz (4)- del precio de las viviendas en 1988 en Madrid (zonas: centro; periferia; corona metropolitana y corona provincial) desglosados según tres partidas: repercusión del suelo (terreno), construcción y gestión. Los valores en pesetas pueden transformarse en \$USA en forma muy aproximada dividiendo por cien. Lo realmente significativo de la Tabla V.1. en pro de la argumentación que aquí se sostiene, es el hecho de la progresiva caída porcentual de la repercusión de la construcción (en la que se engloban los materiales) respecto al precio final de las viviendas en el "Norte", en este caso en Madrid. Desde un $55,55 \%$ en la corona provincial; $50,00 \%$ en la metropolitana; $34,18 \%$ en la periferia hasta un escasísimo $25,64 \%$ en Madrid centro.

Con carácter meramente orientativo, ya que serian muchas y de muy diverso tipo las matizaciones específicas para cada país, se proponen los valores recogidos en la Tabla V.2., que hemos elaborado como desglose del precio final de la vivienda de interés social de dos casos típicos: un país en vías de desarrollo y un país desarrollado. En el primero de los casos, se contemplan dos supuestos, según que dicha vivienda se realice en el sector formal o en el informal.

La mencionada Tabla V.2., intenta plasmar la muy diversa importancia que suponen los distintos factores constituyentes del coste de la vivienda, así como la importancia relativa del factor materiales 
que es marcadamente superior en el sector informal $(64 \%)$ que en el estructurado (43,5\%), y muy superior en el caso de los P.V.D. que en el de los países desarrollados, donde su incidencia llega a caer hasta el $16,7 \%$, ocurriendo para el factor mano de obra el proceso inverso.

Este intento cuantificador confirma la reflexión brasileña recogida más arriba, en el sentido de que el factor tecnológico se encuentra frenado en los países desarrollados por fuentes componentes al margen de los condicionantes de la tecnología, mientras que los P.V.D. muestran una amplia gama de posibilidades para la acción de la tecnología, especialmente tecnología de materiales.

\section{V.3. LA DESCOMPOSICIÓN DEL COSTE DE LA VIVIENDA}

Se intenta el diseccionar la vivienda típica - ¿existe?- de carácter social en sus principales capítulos de obra; al objeto de determinar la repercusión de los materiales en distintos supuestos.

P. Erkelen (5) aporta los datos porcentuales que se recogen en las Tablas V.3. y V.4. como resultados medios de varias realizaciones típicas del conjunto de viviendas de muy bajo coste en diferentes países africanos y asiáticos. La primera proporciona de mayor a menor la repercusión de los diferentes materiales y/o componentes constructivos desde un $26,0 \%$ para los bloques de hormigón hasta un $0,3 \%$ de repercusión del acero estructural. La Tabla V.4. para los mismos casos estudiados agrupa en diez capítulos de obra clásicos -responden con menor o mayor rigor a distintos oficiosaportando su repercusión porcentual: desde un $18,6 \%$ para la superestructura (estructura, cerramientos y tabiquería) hasta un $3,7 \%$ como repercusión del equipamiento de ducha.

El Dr. Minke (6) en un informe-resumen de las actividades del Grupo de Kassel en Guatemala llega a la descomposición siguiente:

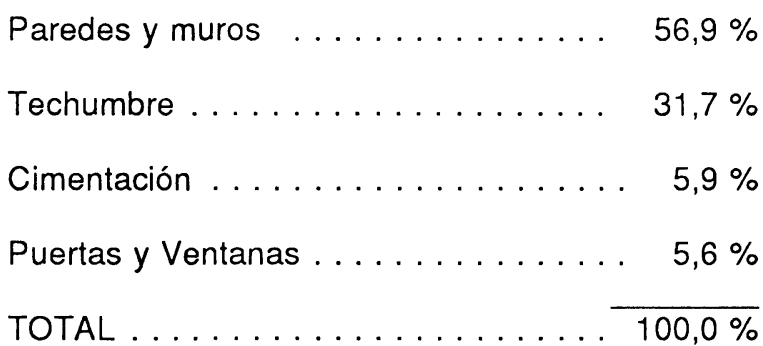

Siguiendo la autorizada opinión del maestro de viviendistas Álvaro Ortega (7): "Los componentes fundamentales de la casa pueden agruparse en las siguientes categorías:

1. La cimentación.

2. El piso.
3. Las paredes.

4. La cubierta.

Ellos componen la cáscara de la casa, mientras que en el interior aparecen:

5. La instalación sanitaria.

6. La instalación eléctrica.

7. La carpintería.

Como ejemplo tendríamos que estos siete factores de costo en una casa económica típica centroamericana con $50 \mathrm{~m}^{2}$ se subdividen así: el $33 \%$ para paredes, el $17 \%$ para el techo, el $13 \%$ para instalaciones sanitarias, el $12 \%$ para la carpintería, el $10 \%$ para la cimentación, el $10 \%$ para los pisos y el $5 \%$ para las instalaciones eléctricas. El cascarón de la casa requiere el $70 \%$ de la inversión total mientras que las instalaciones sólo requieren el $30 \%$. Por otra parte el costo de los materiales representa el $67 \%$, mientras que un $33 \%$ corresponde a la mano de obra.

En el diseño de una casa para usuarios de bajos ingresos el arquitecto tiene que escoger entre áreas disponibles y niveles determinados para ciertas instalaciones y amenidades. Ese profesional debe comprender, cuando está todavía planeando lo que se ha de hacer, el efecto de cada uno de sus diseños en el costo final de la vivienda.

El diseñador debe librarse de sus propios gustos para evitar la producción de niveles más altos que aquellos que pueden ser pagados por los usuarios para quienes diseña. La capacidad de pago de la familia que se ha de albergar, al igual que la cantidad subsidiada, deben ser tenidos en cuenta para determinar la cantidad total de capital que puede ser invertido en la construcción de la vivienda. Cuando se ha establecido ese precio estimativo, el arquitecto debe dar a la familia el máximo de comodidad y amenidades dentro del precio correspondiente. Existen algunos procedimientos para reducir los costos:

\section{A. Reducir el área de la vivienda.}

B. Reducir la calidad de los terminados, instalaciones y amenidades.

\section{Incrementar la producción de los métodos de construcción.}

Para abordar el tema de forma global, partiremos de un trabajo anterior (8) en el que realizamos un desglose porcentual en nueve capítulos -elementos funcionales - de viviendas unifamiliares en España (ver Tabla V.5.).

Los elementos funcionales, concepto que se abordará en profundidad en el Cap. X, estudiados al 
TABLA V.1.

Hipótesis precios vivienda 1988 desglosado por partidas (valor medio ptas. $/ \mathrm{m}^{2}$ )

\begin{tabular}{|l|c|c|c|c|}
\hline & Suelo 1987 & Construcción 1987 & Gestión & Vivienda $\left(\mathrm{ptas} . / \mathrm{m}^{2}\right)$ \\
\hline Madrid Centro & 80.000 & 50.000 & 65.000 & 195.000 \\
Madrid Periferia & 38.000 & 40.000 & 39.000 & 117.000 \\
Corona Metropolitana & 13.000 & 38.000 & 25.000 & 76.000 \\
Corona Provincial & 7.000 & 35.000 & 21.000 & 63.000 \\
\hline
\end{tabular}

TABLA V.2.

Componentes básicos del precio de las viviendas de interés social, expresados en tantos por ciento

\begin{tabular}{|c|c|c|c|c|c|c|c|}
\hline & & & & \multirow{3}{*}{$\begin{array}{l}\text { Suelo } \\
\text { urba- } \\
\text { niza- } \\
\text { ción }\end{array}$} & \multirow{3}{*}{$\begin{array}{l}\text { Finan- } \\
\text { cia- } \\
\text { ción }\end{array}$} & \multirow{3}{*}{ Otros } \\
\hline & & \multicolumn{3}{|c|}{ COSTO DE CONSTRUCCIÓN } & & & \\
\hline & & Total & $\begin{array}{c}\text { Mano } \\
\text { de } \\
\text { obra }\end{array}$ & $\begin{array}{l}\text { Mate- } \\
\text { riales }\end{array}$ & & & \\
\hline \multirow{2}{*}{ P.V.D.* } & SECTOR "FORMAL" & $65 \%$ (1/3 m.o. + 2/3 mat. $)$ & $22 \%$ & $43 \%$ & $20 \%$ & $10 \%$ & $5 \%$ \\
\hline & SECTOR "INFORMAL" & $80 \%$ (1/5 m.o. + 4/5 mat.) & $16 \%$ & $64 \%$ & $5 \%$ & $10 \%$ & $5 \%$ \\
\hline \multicolumn{2}{|c|}{ PAÍSES DESARROLLADOS } & $50 \%(2 / 3$ m.o. + 1/3 mat. $)$ & $33,3 \%$ & $16,7 \%$ & $25 \%$ & $15 \%$ & $10 \%$ \\
\hline
\end{tabular}

*P.V.D. = Países en vías de desarrollo.

TABLA V.3.

Materiales ordenados según el grado

de repercusión del coste de construcción

\begin{tabular}{|l|c|}
\hline \multicolumn{1}{|c|}{ Material } & $\begin{array}{c}\text { \% del coste del } \\
\text { Material }\end{array}$ \\
\hline Bloques de Hormigón & 26,0 \\
Material de Cubierta & 14,2 \\
Cemento & 14,2 \\
Instalación Sanitaria & 10,9 \\
Aparatos Sanitarios & 6,9 \\
Puertas & 5,2 \\
Ferretería & 4,1 \\
Arena & 3,6 \\
Madera & 2,9 \\
Áridos & 2,8 \\
Fontanería & 2,2 \\
Pintura & 1,8 \\
Accesorios & 1,8 \\
Vidrios & 1,7 \\
Ventanas & 1,4 \\
Acero de Refuerzo & 0,3 \\
TOTAL & 100,0 \\
\hline
\end{tabular}

TABLA V.4.

Orden de magnitud de la repercusión de los elementos funcionales

\begin{tabular}{|l|c|}
\hline \multicolumn{1}{|c|}{ Elementos } & \% del Total \\
\hline Superestructura & 18,6 \\
Cubierta & 15,4 \\
Acabados & 13,1 \\
Cimentaciones & 10,2 \\
Puertas y Ventanas & 9,6 \\
Equipo de Cocina & 9,2 \\
Soleras y Suelo & 7,2 \\
Fregadero & 6,7 \\
W.C. & 6,3 \\
Ducha & 3,7 \\
TOTAL & 100,0 \\
\hline
\end{tabular}


objeto de evaluar la relativa participación porcentual de los mismos en el coste final de construcción fueron:

1. Excavación, movimiento de tierras y cimentación.

2. Cubierta (incluyendo la estructura portante de la misma).

3. Plano de contacto: solera y piso totalmente acabados.

4. Estructura (no incluye muros portantes).

5. Equipo de la vivienda: agua, gas, electricidad, calefacción, armarios,...

6. Acceso, porche y entrada.

7. Elementos verticales interiores incluida carpintería de huecos y acabados.

8. Elementos verticales exteriores incluida carpintería de huecos y acabados.

9. Locales de cocina y baño incluyendo sanitarios.

Siguiendo idénticos criterios y en base a diferentes trabajos y datos acumulados, se han estimado los diferentes valores porcentuales de repercusión para los mismos nueve capítulos de obra en el caso de una vivienda de bajo coste en un hipotético caso "medio" en América Latina.

La Tabla V.5. es, pues, resumen de un conjunto de resultados de muy diversa procedencia: la primera columna recoge valores resultantes del trabajo antes mencionado del autor; la columna tercera son valores estimados en base a datos muy diversos de distinta procedencia y las columnas segunda y cuarta responde a una propuesta meramente intuitiva consistente en aplicar los coeficientes $2 / 5$ y $2 / 3$ a los valores de las columnas primera y tercera respectivamente. Obviamente, el conjunto de datos de esta Tabla, tiene validez en este contexto y exclusivamente con carácter metodológico.

Los datos de la Tabla V.5. se han vertido en la Fig. V.1., para lo que se ha utilizado una escala única de ordenadas (izquierda) para los valores porcentuales de la repercusión de los nueve capítulos de obra y una escala doble (ordenadas de la derecha) para las repercusiones porcentuales de los materiales en el caso de España y Latinoamérica respectivamente.

Aun teniendo muy presente el origen de los datos, la Fig. V.1. quiere ser resumen de una idea obsesiva del autor: la importancia de los materiales, el tipo de materiales y la repercusión sobre el coste final, son muy distintos según que hablemos del Norte o del Sur.

\section{V.4. EL CEMENTO: MATERIAL EMBLEMÁTICO EN AMÉRICA LATINA}

Dada la importancia del cemento en la construcción de viviendas, parece oportuno dedicarle a este material un Apartado monográfico. No debe olvidarse que una vivienda completa de unos $60 \mathrm{~m}^{2}$, en el sector formal, a base de elementos de hormigón (prefabricado o no) puede suponer un consumo de 6-8 t de cemento, y que difícilmente se baja de los $15 \mathrm{~kg}$ de consumo de cemento portland para realizar un metro cuadrado de elemento construido.

Otro dato que debe tenerse presente al abordar la importancia del cemento es el de su repercusión sobre el coste total de la vivienda social, ver Apartado V.1.

Por todo lo dicho, no es de extrañar que se genere (en los países del Norte) una importante actividad de investigación y desarrollo tecnológico en torno del cemento, actividad que puede esquematizarse en varias direcciones:

a. Optimizar los procesos de producción de cemento, especialmente en el sentido del ahorro energético en su fabricación.

b. Tendencia a la búsqueda de minifactorías rentables y competitivas.

c. Utilización racional del cemento: hormigones de baja dosificación, empleo de adiciones, estudios de granulometría, etc.

d. Búsqueda de materiales sustitutivos/complementarios del cemento: puzolanas naturales, cales, desechos agroindustriales, etc.

Por su interés, se reproduce seguidamente un aspecto del trabajo de CEPAL sobre "La Industria del Cemento en la Región Latinoamericana y del Caribe: Evolución, Situación Actual y Perspectivas de Desarrollo Tecnológico" (9).

"En América Latina y el Caribe se encuentran actualmente en funcionamiento aproximadamente unas 170 plantas productoras de cemento. De ellas, 150 están instaladas en países miembros de la Asociación Latinoamericana de Integración (ALADI) y del Mercado Común Centroamericano (MCCA). En cuanto a las 20 restantes, 13 se encuentran en los países del Caribe de habla española (entre ellas 7 en Cuba) y 7 en Suriname y el resto del Caribe.

La producción conjunta de cemento de 22 países de América Latina y el Caribe alcanzó en 1977, 58 millones de toneladas y aumentó a 75 millones de toneladas en 1981, lo que representa una tasa media de crecimiento anual de $6,6 \%$ en este período. Cabe señalar que casi un $70 \%$ de esta producción, durante todo el lapso mencionado, corresponde sólo a tres países: Argentina, Brasil y México. Otras proporciones de cierta importancia 
TABLA V.5.

\begin{tabular}{|c|c|c|c|c|}
\hline \multirow[b]{2}{*}{$\begin{array}{c}\text { Viviendas de una planta. } \\
\text { Capitulos de obra o elementos funcionales } \\
\text { totalmente acabados }\end{array}$} & \multicolumn{2}{|c|}{ Vivienda Unifamiliar en España } & \multicolumn{2}{|c|}{$\begin{array}{l}\text { Vivienda de Muy Bajo Coste } \\
\text { en América Latina }\end{array}$} \\
\hline & $\begin{array}{l}\text { Repercusión } \\
\text { Total } \\
\text { (en \%) }\end{array}$ & $\begin{array}{c}\text { Repercusión } \\
\text { de los } \\
\text { materiales } \\
2 / 5 \text { total } \\
\text { (en \%) }\end{array}$ & $\begin{array}{l}\text { Repercusión } \\
\text { Total } \\
\text { (estimada) } \\
\text { (en \%) }\end{array}$ & $\begin{array}{c}\text { Repercusión } \\
\text { de los } \\
\text { materiales } \\
2 / 3 \text { total } \\
\text { (en } \% \text { ) }\end{array}$ \\
\hline 1. Excavaciones/cimentación & 8,50 & 3,4 & 8 & 5,3 \\
\hline 2. Cubierta & 7,30 & 2,9 & 15 & 10,0 \\
\hline 3. Solera y suelo acabado (plano de contacto) & 15,00 & 6,0 & 5 & 3,3 \\
\hline 4. Estructura & 7,30 & 2,9 & 8 & 5,3 \\
\hline 5. Equipo de la vivienda & 16,30 & 6,5 & 4 & 2,7 \\
\hline 6. Acceso, Vestíbulo & 8,40 & 3,4 & 1 & 0,7 \\
\hline 7. Elementos verticales interiore (tabiques) & 8,30 & 3,4 & 16 & 10,6 \\
\hline 8. Elementos verticales exteriores (fachadas) & 23,90 & 9,6 & 36 & 24,0 \\
\hline 9. Cocina/baño & 6,00 & 2,4 & 4 & 2,7 \\
\hline TOTALES & 100,00 & 40,5 & 100 & 64,6 \\
\hline
\end{tabular}

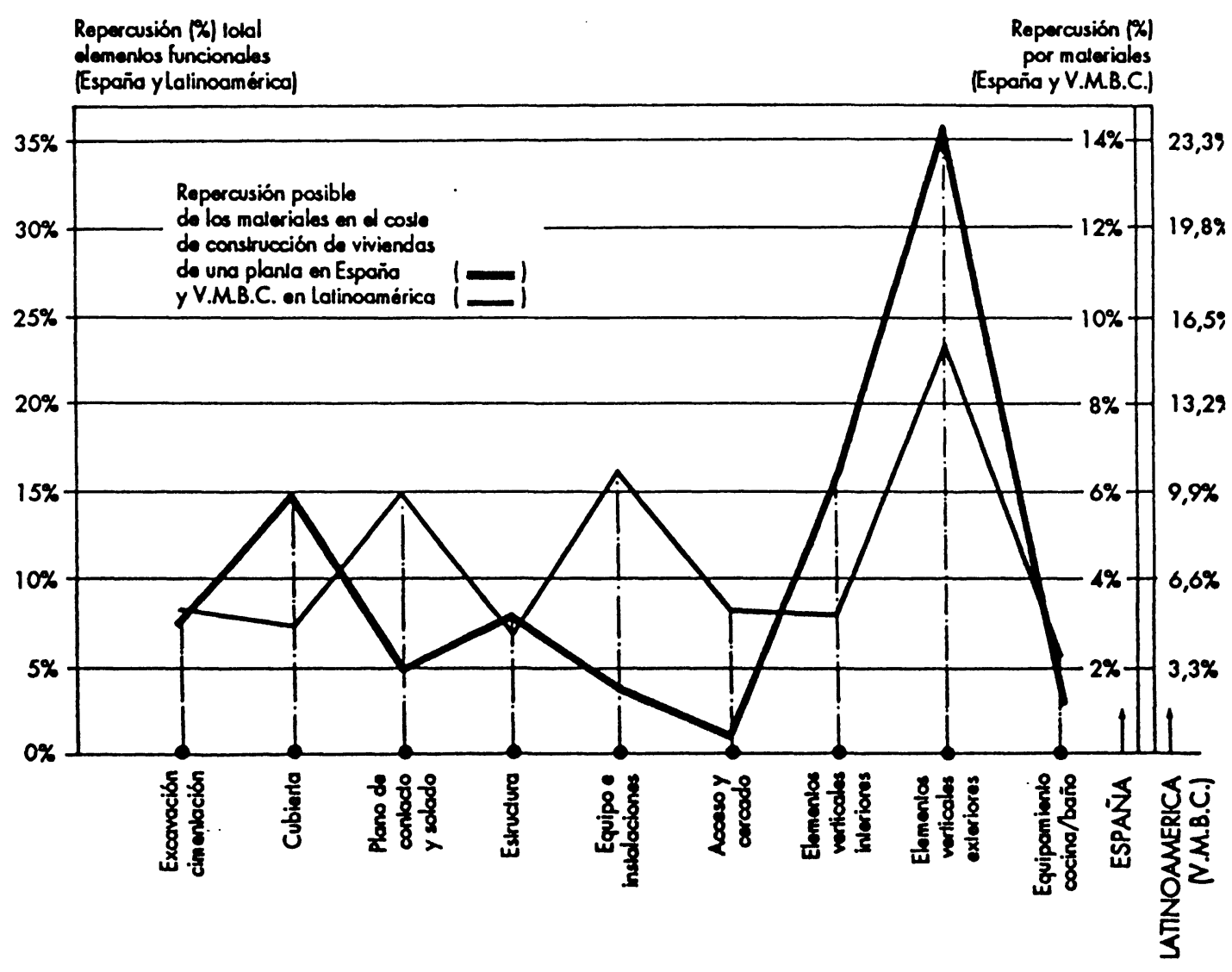

Fig. V.1. 
dentro del total son las de Colombia, Cuba, Chile, Ecuador, Perú y Venezuela, cuyas producciones, en general, aumentan sostenidamente en esos años. Sin embargo, la tendencia se interrumpe en 1982 en los 22 países considerados, principalmente como un efecto de la situación recesiva de las economías; la disminución es general, con excepción de Colombia, Ecuador, México, Perú y Venezuela. En el período 1979-1982 cabe destacar los mayores crecimientos de México $(8,1 \%)$, Perú $(5,4 \%)$ y Venezuela $(7,8 \%)$. (Ver Tabla V.6.).

TABLA V.6.

América latina capacidad instalada de la industria del cemento por países, 1979

\begin{tabular}{|l|c|c|}
\hline Pais & $\begin{array}{c}\text { Capacidad } \\
\text { instalada } \\
\text { (miles de } \\
\text { toneladas } \\
\text { anuales) }\end{array}$ & $\begin{array}{c}\text { Parti- } \\
\text { cipación } \\
\text { relativa } \\
(\%)\end{array}$ \\
\hline Argentina & 8.855 & 10,7 \\
Bolivia & 610 & 0,7 \\
Brasil & 27.945 & 33,7 \\
Colombia & 5.470 & 6,6 \\
Costa Rica & 1.050 & 1,3 \\
Cuba & 6.996 & 7,2 \\
Chile & 1.750 & 2,1 \\
Ecuador & 1.482 & 1,8 \\
El Salvador & 562 & 0,7 \\
Guatemala & 886 & 1,1 \\
Honduras & 400 & 0,5 \\
México & 16.629 & 20,1 \\
Nicaragua & 320 & 0,4 \\
Panamá & 600 & 0,7 \\
Paraguay & 216 & 0,3 \\
Perú & 3.170 & 3,8 \\
Rep. Dominicana & 1.523 & 1,8 \\
Uruguay & 766 & 0,9 \\
Venezuela & 4.612 & 5,6 \\
TOTAL & 82.864 & 100,0 \\
\hline & & \\
\hline
\end{tabular}

La industria latinoamericana del cemento presenta una marcada concentración de la propiedad industrial. Al respecto, puede señalarse que, de acuerdo con la información disponible, en Brasil son solamente tres los grupos empresariales que controlan la mitad de la producción nacional. En México, tres grupos disponen de dos tercios de la capacidad instalada del país $y$, en Colombia, unas pocas empresas del rubro son propietarias de una proporción considerable del capital de otras. Lo mismo se observa en Argentina y Venezuela, donde aparentemente el fenómeno se ha producido debido a que las empresas pioneras han impulsado el desarrollo de nuevas empresas en el sector. En el resto de los países latino-americanos, la concentración probablemente responde al reducido tamaño de los mercados nacionales, sólo suficientes para unas pocas plantas.

En América Latina, la industria del cemento está en su mayor parte en poder de propietarios nacionales independientes, aunque todavía existen filiales de empresas transnacionales, como en Argentina, Chile, Uruguay (en este último caso, se trata de inversiones que datan de comienzos de siglo). Al menos en Argentina y Uruguay, las empresas transnacionales no tienen una participación importante en el sector. En México, la inversión extranjera en este rubro industrial corresponde solamente a un tercio de la capacidad instalada total en el país, y está sujeta a controles gubernamentales" (9).

\section{V.5. LA INVESTIGACIÓN NECESARIA EN EL CAMPO DE LOS MATERIALES}

Con parecido título al de la cabecera, el Dr. Gyula Sebestyen (10), Secretario General del Consejo Internacional de la Edificación (C.I.B.) presentó por encargo de ONUDI, un trabajo monográfico preparatorio de la "Primera Consulta sobre la Industria de los Materiales de Construcción".

Pese a que no es práctico el definir prioridades en materia de investigación sin situarlas en un momento y geografía determinado, sí puede ser de interés, en el contexto de este trabajo, contar con lo que son actualmente líneas maestras o globales admitidas entre las grandes prioridades. En este sentido, Sebestyen apuntan las siguientes:

a. Utilización de materiales y subproductos locales (nacionales); conservación de recursos y energía.

b. Aumento de la durabilidad y la pirorresistencia de los materiales de construcción autóctonos.

c. Tecnologías apropiadas; aspectos económicos de las investigaciones y desarrollos tecnológicos.

d. Aplicación de técnicas de control de calidad para el mejor uso de los recursos.

e. Conservación de los recursos naturales y energéticos.

Como concreción de las cinco prioridades absolutas enumeradas, Sebestyen señala las siguientes prioridades en materia de investigación en el campo de los materiales de construcción básicos:

1. Tierra (arcilla, adobe, laterita). 
2. Cemento, cal, yeso y puzolana:

- Construcción de fábricas sencillas de baja o media capacidad.

- Empleo de materias primas y subproductos locales.

- Empleo de combustibles locales para la calcinación.

3. Hormigón, ferrocemento, ladrillos, bloques, elementos premoldeados.

4. Madera, bambú, y otros productos y subproductos vegetales.

5. Relaciones entre las investigaciones sobre los materiales y la construcción.

Cabe prever con un grado suficiente de certeza que durante los próximos decenios aumentarán las necesidades de materiales de construcción de los países en desarrollo. Hay que esforzarse porque el mayor porcentaje posible de la población continúe utilizando materiales locales, pero el crecimiento de la población y el inevitable proceso de urbanización incrementarán sustancialmente la demanda. Los recursos locales están disminuyendo en algunas zonas y será necesario utilizar mejor aquello de que se dispone. Habrá que proporcionar materiales de construcción a más y más familias. El desarrollo de la industria, las ciudades y las aldeas aumentará la demanda de materiales de construcción.

Ningún país puede depender en exceso de las importaciones de materiales de construcción. Cada país tiene ciertos tipos de recursos propios que pueden emplearse para la producción de materiales de construcción. Esta producción debe desarrollarse tanto en los subsectores informal como en el formal. Se necesitan inversiones de capital privado y público para alcanzar niveles superiores en la producción de materiales de construcción.

La selección de las prioridades en materia de investigación debe tener en cuenta ciertas tendencias generales (incremento de la durabilidad, etc.), así como las condiciones locales. La investigación debe orientarse hacia la determinación de los campos en los que sean factibles los mayores beneficios y aquellos en los que la sustitución de importaciones o compra de know-how pueden constituir la opción más eficaz.

La investigación sobre los materiales de construcción y la industria de la construcción debería efectuarse en prácticamente todos los países del Area, sin que ello diese como resultado tendencias hacia una investigación autárquica. La eficiencia de la investigación puede aumentarse mediante la cooperación internacional Sur-Sur.

\section{V.5.1 La búsqueda de sustitutivos a los con- glomerantes tipo portland}

En el contexto de la investigación necesaria y presentando el cemento como material emblemáti$\mathrm{co}$, parece oportuno el detenerse en la necesidad de profundizar en la búsqueda de sustitutivos y complementarios al mismo. Nuestro acercamiento profesional al tema, se enmarca en el siguiente contexto:

a. No se trata de buscar la sustitución utópica del empleo del cemento, sino su lógica complementación. Los cementos tipo portland tiene asegurado un futuro estable y duradero.

b. Si en la búsqueda del empleo de subproductos agroindustriales y productos naturales como conglomerantes, se emplease a nivel mundial un esfuerzo semejante (en medios humanos y recursos) al que se dedica al cemento, los resultados prácticos serían muy diferentes.

Bajo este marco conceptual, se presentan algunos datos que justifican la búsqueda de otros conglomerantes:

a. La búsqueda de conglomerantes autóctonos sustitutivos-complementarios de la importación de cementos tipo portland para determinadas aplicaciones, es una línea de investigación prioritaria a nivel internacional propiciada por numerosas instituciones internacionales: ONUDI; UNCH-HABITAT; C.I.B.; Programa CYTED.D; etc.

b. En 1982 el consumo mundial de cemento se calculaba en 860 millones de toneladas y las estimaciones para el año 2000 preven un consumo de 1.300 millones de toneladas.

En la actualidad el consumo de cemento se encuentra muy concentrado en los países industrializados. Según Boubekeur, EE.UU. Japón, República Federal de Alemania, Francia e Italia absorben el $30 \%$ de la producción mundial, mientras que en el año 2000 el consumo mundial preentará la tendencia siguiente:

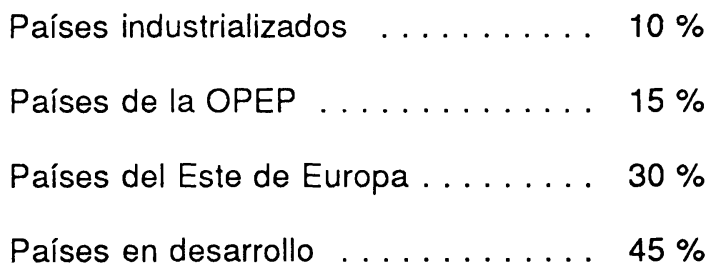

c. Al factor de los fuertes contrastes que presenta el tema de la distribución heterogénea de la producción hay que añadir el de los costes.

A título de comparación, señala CEPAL que la tonelada de cemento producida en Francia costaba 264 francos en 1980, mientras que el 
precio del cemento en Senegal era de $580 \mathrm{FF}$, en Ghana de 900 FF y llegaba a 953 FF, en Nairobi.

Por otra parte, el precio del cemento que carga el distribuidor puede duplicarse a una distancia superior a 200 kilómetros.

En ciertos estudios se señala incluso que el precio de la tonelada de cemento puesta en fábrica puede multiplicarse por diez en el curso de la distribución. Entre los factores que pueden contribuir a aumentar el precio figuran la dispersión de los mercados y las difíciles condiciones de transporte, almacenamiento y conservación del producto.

d. En el campo de la investigación sobre esta temática, parece de interés resaltar que la fabricación de cemento requiere mayor densidad de capital que la de cal y puzolana. Por ello, es importante sustituir donde sea factible, aunque sea parcialmente, el cemento por cal y puzolana. Este objetivo también puede lograrse mediante la fabricación de cementos mezclados, en los que parte del cemento se sustituye por puzolana natural o por subproductos industriales (cenizas volantes, escorias, arena) con propiedades hidráulicas. Si la infraestructura de carreteras y ferrocarriles es inadecuada y la capacidad del sistema de transporte insuficiente, puede producirse una escasez local de cemento y otros materiales de construcción. Es preciso determinar cuáles son las materias primas apropiadas, así como estudiar la fabricación y el empleo de cemento en plantas locales (subregionales). Estas tareas son de alta prioridad para los investigadores de los materiales de construcción.
De acuerdo con lo anterior, parece oportuno concluir afirmando que no siempre -y muy especialmente en algunos países o zonas del Áreaconviene emplear cementos portland para todo tipo de obras. Una gestión eficaz del empleo de los conglomerantes debería contemplar:

1. Utilización del cemento portland para obras de responsabilidad.

2. Fomento de la producción de otros conglomerantes: cal, puzolanas,... para obras de pocas necesidades resistentes (viviendas de una planta).

3. Impulso de la investigación de conglomerantes autóctonos o derivados de subproductos agroindustriales.

\section{V.5.2. Tres trabajos reseñables}

De forma muy escueta y como simple enumeraciónde ideas sobre la investigación necesaria, nos permitimos seleccionar tres trabajos que apuntan a este sentido.

La Dirección General de Carreteras de Francia elaboró la Tabla V.7. en la que se recoge un listado de subproductos utilizables en la construcción de carreteras que califica según tres familias o clases:

- Clase I: directamente utilizables.

- Clase II: utilizables tras sufrir procesos físicos sencillos.

- Clase III: utilizables tras someterse a procesos de carácter físico-químicos de cierta consideración.

TABLA V.7.

Evaluación global de desechos y subproductos en construcción de carreteras

\begin{tabular}{|l|l|l|}
\hline \multicolumn{1}{|c|}{ CLASE I } & \multicolumn{1}{|c|}{ CLASE II } & \multicolumn{1}{c|}{ CLASE III } \\
\hline \begin{tabular}{l|l} 
Escoria de alto horno \\
Cenizas volantes
\end{tabular} & $\begin{array}{l}\text { Escoria de fosfato } \\
\text { Escoria de cobre } \\
\text { Azufre }\end{array}$ & $\begin{array}{l}\text { Ganga de oro } \\
\text { Ganga de cobre }\end{array}$ \\
Escoria de acerería & Áridos de mina & Ganga de plomo-zinc \\
Escoria de caldera & Desechos de pizarra & Carbonilla de cok \\
Cenizas de tolva & Cáscaras y aserrín & Aceros de fundición \\
Escoria de niquel & Ganga de espato-fluor & Cerámicas y desechos refractarios \\
Materiales de demolición & Neumáticos y caucho & Resina \\
Esquistos de hulla & Escoria de zinc & Lignina \\
Ganga de taconita & Escoria de plomo & Desechos de minas de sales de potasio \\
Arena de caolín & Residuos de incinerador & Kiessaffrand \\
Ganga de pirita & Desechos de vidrio \\
Residuos de pyronisina & Desechos de plástico \\
\hline
\end{tabular}


El segundo aspecto que en esta misma dirección nos interesa resaltar lo constituye la Figura V.2., que tomamos del trabajo de tesis doctoral de Isabel Sánchez (11) sobre "Clasificación de Materiales con Capacidad Puzolánica" que permite visualizar la amplia gama de productos naturales y artificiales que en mayor o menor medida disponen de esa característica.

Para finalizar, y también en forma de Tabla V.8. recogemos las siete líneas de trabajo de investigación aplicada propuestas por el autor, como algunas de las más necesarias de abordar con posibilidad de éxito en el campo de las "Tecnologías para Viviendas de Interés Social en Latinoamérica" y que de hecho, constituyen el programa de actuación a medio plazo del Programa Ciencia y Tecnología para el Desarrollo (CYTED.D) en su Subprograma XIV: Tecnología para Vivienda de Interés Social (12).

TABLA V.8.

Propuesta de estructuración del subprograma XIV: "Tecnología para viviendas de interés social" del programa CYTED.D

1. Tecnologías para la construcción a base de madera: caracterización de maderas; optimización de su empleo (durabilidad y pirorresistencia), soluciones constructivas a base de componentes de madera.

2. Elementos, componentes y sistemas constructivos para su empleo en viviendas crecederas y/o mejorables mediante autoconstrucción o ayuda mutua: en situación urbana; en ambiente rural.

3. Tecnologías para la construcción a base de tierra: optimización del empleo de la tierra (resistencia, comportamiento ante la humedad, tratamiento de parámetros,...). Soluciones específicas constructivas y estructurales para su utilización en zonas de alta sismicidad.

4. Desarrollo, experimentación y evaluación de tecnologias apropiadas para la mejora y consolidación de asentamientos populares espontáneos. Construcción y valorización de soluciones habitacionales y servicios comunitarios.

5. Desarrollo de acuerdos de compatibilización, coordinación y de prestaciones funcionales de elementos; subsistemas y sistemas constructivos para viviendas de interés social, mediante tecnologia libre a base de elementos prefabricados y utilizando la capacidad ya instalada en el Área.

6. Soluciones de cubiertas a base de elementos ligeros realizados prioritariamente mediante materiales y/o subproductos locales capaces de sustituir las placas usuales (asbesto-cemento o zinc).

7. Optimización de procesos de producción y de empleo del cemento portland. Caracterización tecnológica de adiciones y de hormigones a base de cementos con adiciones.
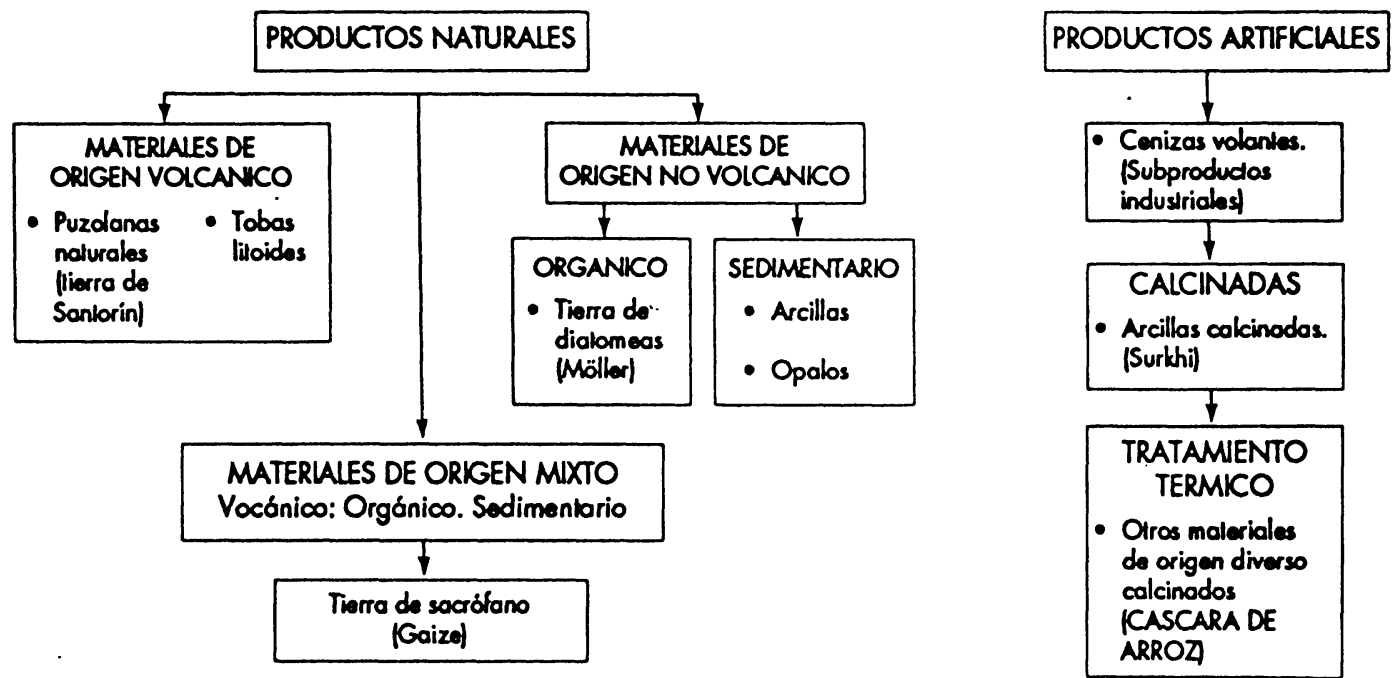

Fig. V.2.-Clasificación de materiales con capacidad puzolánica. 


\section{BIBLIOGRAFIA}

(1) H. HOUBEN: "L'habitat èconomique dans les pays en developpment: materiaux, techniques de construction, composants". Plan Construction. París 1984, Volumen 2, Págs. 53 y 60.

(2) E. RAMACHANDRAN: "Discurso de apertura en la Primera Consulta sobre materias de construcción de ONUDI". Atenas, mayo 1985.

(3) Consehlho Nacional de Desenvolvimento científico y Tecnológico. "Programa integrado para desenvolvimento de assentamentos humanos", 95 págs. Brasilia, septiembre, 1985.

(4) Revista ALFOZ, n. 65. Monografía n. 47. Madrid, 1987.

(5) P. ERKELEN: "The economics of building materials research". Congreso "Appropriate building for low cost housing". CIB-RILEM. Nairobi, 1983, págs. 321 a 329.

(6) MINKE GERNOT: "Sistema de vivienda de bajo costo para Guatemala". Laboratorio de Construcciones Experimentales. Universidad de Kacsel, junio 1980, 70 páginas.

(7) ALVARO ORTEGA: "Alvaro Ortega: Prearquitectura del Bienestar". Colección Somosur. Edit. Escala. Bogotá. Colombia 1989.

(8) JULIAN SALAS y S. PÉREZ ARROYO: "Estudio tipológico de viviendas en España y su descomposición de costes". Trabajo mecanografiado. Madrid, 1977.

(9) CEPAL: "La industria del cemento en la región latinoamericana y del Caribe: evolución, situación actual y perspectiva del desarrollo tecnológico". Manual.

(10) SEBESTYEN GYULA: "Prioridades en materia de investigación para las industrias de los materiales de construcción de los países en desarrollo". ONUDI, ID/ING/425/1. 61 págs. Viena (Austria), 1984.

(11) ISABEL SÁNCHEZ: "Clasificación de Materiales con Capacidad Puzolánica". Trabajo mecanografiado. Instituto E. Torroja. Madrid 1988.

(12) TED.D: "Tecnologias para Viviendas de Interés Social". Cuatro tomos Mecanografiado. Madrid, 1987.

\section{publicación del ICCET/CSIC INSPECCION DE OBRAS DAÑADAS
POR CORROSION DE ARMADURAS}

El presente Manual va dirigido principalmente a técnicos especializados y laboratorios que tienen que intervenir en el dictamen de la situación de deterioro de estructuras de hormigón armado dañadas por corrosión de armaduras.

Comienza con un resumen recordatorio de los factores principales a los que se pueden deber los daños prematuros por corrosión de armaduras, para seguir con algunas indicaciones de cómo se deben realizar las inspecciones, y de los ensayos y la metodologia que se recomienda realizar para poder dictaminar con precisión las causas de daño.

A continuación se hacen una serie de comentarios sobre la vida residual de estructuras dañadas, sobre el riesgo de corrosión futura, el seguimiento necesario de una estructura reparada y una breve enumeración de métodos de reparación y consideraciones básicas a tener en cuenta en la recomendación de un determinado método. Se aporta una breve relación bibliográfica.

Finalmente se incluyen en forma de ficha la descripción de algunos casos de corrosión de armaduras detectados en nuestro país. manual

inspección de obras dañadas por corrosión de armaduras

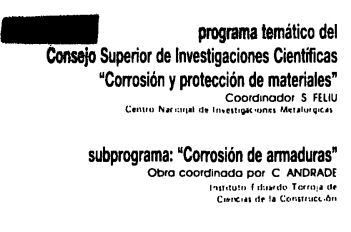

